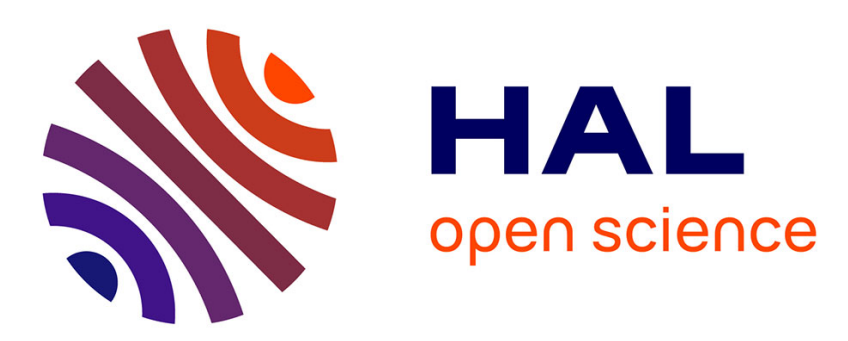

\title{
Attainability by simply connected domains of optimal bounds for the polarization tensor
}

Habib Ammari, Yves Capdeboscq, Hyeonbae Kang, Eunjoo Kim, Mikyoung Lim

\section{- To cite this version:}

Habib Ammari, Yves Capdeboscq, Hyeonbae Kang, Eunjoo Kim, Mikyoung Lim. Attainability by simply connected domains of optimal bounds for the polarization tensor. European Journal of Applied Mathematics, 2006, 17 (2), pp.201-219. 10.1017/S0956792506006541 . hal-02083815

\section{HAL Id: hal-02083815 https://hal.science/hal-02083815}

Submitted on 10 Feb 2021

HAL is a multi-disciplinary open access archive for the deposit and dissemination of scientific research documents, whether they are published or not. The documents may come from teaching and research institutions in France or abroad, or from public or private research centers.
L'archive ouverte pluridisciplinaire HAL, est destinée au dépôt et à la diffusion de documents scientifiques de niveau recherche, publiés ou non, émanant des établissements d'enseignement et de recherche français ou étrangers, des laboratoires publics ou privés. 


\title{
ATTAINABILITY BY SIMPLY CONNECTED DOMAINS OF OPTIMAL BOUNDS FOR THE POLARIZATION TENSOR
}

\author{
HABIB AMMARI ${ }^{1}$, YVES CAPDEBOSCQ ${ }^{2}$, HYEONBAE KANG ${ }^{3}$, EUNJOO KIM ${ }^{1}$, \\ AND MIKYOUNG LIM ${ }^{1}$
}

\begin{abstract}
The notion of polarization tensor is employed for the derivation of the leading-order boundary perturbations in the steady-state voltage potentials that are due to the presence of conductivity inclusions of small diameter. Recently, Capdeboscq and Vogelius obtained optimal bounds of HashinShtrikman type for the trace of the polarization tensor, showing that every pair satisfying these optimal bounds arises as the eigenvalues of a polarization tensor associated with a coated ellipse. In this paper, we give numerical evidence of the fact that the set of possible polarization tensor eigenvalue pairs can also be obtained using simply connected domains. Our numerical computations are based on a boundary integral method.
\end{abstract}

\section{INTRODUCTION}

This paper is concerned with the notion of polarization tensor (PT) associated with a bounded Lipschitz domain and an isotropic constant conductivity. The notion of PT appeared in problems of potential theory related to certain questions arising in hydrodynamics, in electrostatics, and in low-frequency scattering; see $[22,21,14]$. The PT is a key mathematical concept in efficiently imaging small conductivity inclusions from boundary measurements and also in calculating effective electrical properties of composite materials consisting of inclusions of one material of known shape embedded homogeneously into a continuous matrix of another having electrical properties different from its own. It is now known that the leading-order term in the boundary perturbations due to the presence of an inclusion inside a conductor as well as in the asymptotic expansion of the effective conductivity of a dilute composite material in terms of the volume fraction of the inclusions can be expressed by means of the PT of the inclusions shape; see $[13,8,11,1,4,6,15,20,16]$. The asymptotic expansion of the effective conductivity is motivated by the practically important inverse problem of determining the volume fraction of a suspension of complicated shaped particles from boundary measurements of voltage potentials. Therefore, in both applications, it is important from an imaging point of view to precisely characterize the class of PTs and

\footnotetext{
${ }^{11}$ Centre de Mathématiques Appliquées, CNRS UMR 7641 and Ecole Polytechnique, 91128 Palaiseau Cedex, France

email: ammari@cmapx.polytechnique.fr, eunjoo@cmapx.polytechnique.fr, mklim@cmapx.polytechnique.fr

${ }^{2}$ Département de Mathématiques, Université de Versailles Saint-Quentin-en-Yvelines, Bât. Fermat, 45 Avenue des Etats-Unis, 78035 Versailles Cedex, France

email: Yves.Capdeboscq@math.uvsq.fr

${ }^{3}$ Department of Mathematical Sciences and RIM, Seoul National University, Seoul 151-747, Korea

email: hkang@math.snu.ac.kr
} 
extract from the (detected) PT some important information, such as the size and the orientation of the inclusion. Indeed, it is important to obtain bounds for the matrix-elements of the PT since a better bound for the PT yields a better estimate for the size of the inclusion; see $[11,1]$.

Recently, based on variational techniques originally described in [17], Capdeboscq and Vogelius [11] obtained geometry independent optimal bounds of HashinShtrikman (HS) type for the trace of the PT. These bounds had already been derived, by an alternative approach, by Lipton [19]. Throughout this paper, these bounds are called the HS bounds. To mathematically state the main estimates provided in [11], let $M$ denote the PT associated with the bounded domain $D \subset \mathbb{R}^{d}$ whose volume $|D|=1$ and the constant conductivity $0<k \neq 1<+\infty$. Then, for $d=2,3$, these bounds are as follows:

$$
\operatorname{Trace}(M) \leq(k-1)\left(d-1+\frac{1}{k}\right),
$$

and

$$
\operatorname{Trace}\left(M^{-1}\right) \leq \frac{d-1+k}{k-1} .
$$

In particular, in the two-dimensional case, if $\lambda_{1}$ and $\lambda_{2}$ are two eigenvalues of $M$, then

$$
\lambda_{1}+\lambda_{2} \leq \frac{(k-1)(k+1)}{k},
$$

and

$$
\frac{1}{\lambda_{1}}+\frac{1}{\lambda_{2}} \leq \frac{k+1}{k-1} .
$$

Figure 1 shows these bounds graphically, where the region $A$ is the upper part of the HS bounds, namely the set of all $\left(\lambda_{1}, \lambda_{2}\right)$ satisfying

$$
\lambda_{1}+\lambda_{2}<\frac{(k-1)(k+1)}{k}, \quad \frac{1}{\lambda_{1}}+\frac{1}{\lambda_{2}} \leq \frac{k+1}{k-1}, \quad \lambda_{1} \leq \lambda_{2},
$$

and the region $B$ is the set of all $\left(\lambda_{1}, \lambda_{2}\right)$ satisfying

$$
\lambda_{1}+\lambda_{2}<\frac{(k-1)(k+1)}{k}, \quad \frac{1}{\lambda_{1}}+\frac{1}{\lambda_{2}} \leq \frac{k+1}{k-1}, \quad \lambda_{1} \geq \lambda_{2} .
$$

The question we deal with in this paper is whether each point inside the HS bounds is a pair of eigenvalues of a PT associated with a domain of unit area in $\mathbb{R}^{2}$. This question was positively answered by Capdeboscq and Vogelius in [12]. These authors showed that each point inside the HS bounds is attained as a PT associated with a coated ellipse, or a washer of elliptic shape. In fact, every point on the lower bound $1 / \lambda_{1}+1 / \lambda_{2}=(k+1) /(k-1)$ corresponds to an ellipse, and as ellipses get thinner, corresponding points on the lower bound move to the upper or lower corner. If we start from an ellipse corresponding to a point on the lower bound, and make confocal washers of elliptic shape, then corresponding points move toward the upper bound following a certain curve as the washers get thinner and larger. These curves make foliations and cover all regions inside the bounds except the upper bound.

Our goal in this paper is to give numerical evidence of the fact that this optimal set can also be obtained using simply connected domains. Our approach is to compute numerically the polarization tensors corresponding to a radial interpolation 


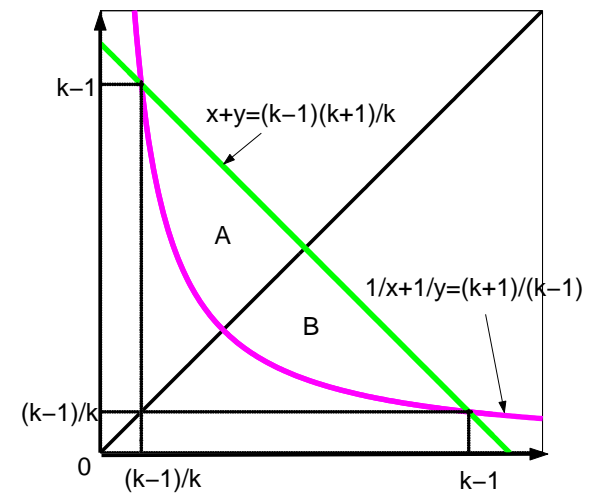

Figure 1. The optimal bounds for the polarization tensor in $\mathbb{R}^{2}$.

between ellipses of unit area and crosses with right angles, equal legs, and smooth corners. It turns out that if we start from the unit disk and vary the domain to make a thin and long cross as shown in Figure 2, the corresponding eigenvalues move from the intersection point of the lower hyperbola and the line $\lambda_{1}=\lambda_{2}$ toward the intersection point of the upper bound and the line $\lambda_{1}=\lambda_{2}$ following the line $\lambda_{1}=\lambda_{2}$. Note that the intersection point of the lower hyperbola and the line $\lambda_{1}=\lambda_{2}$ is the pair of eigenvalues of the PT associated with the unit disk. We also note that the cross-shaped domain in Figure 2 is invariant under rotation by $\pi / 2$, and hence the corresponding PT is of the form $\lambda I$ for some $\lambda$ where $I$ is the $2 \times 2$ identity matrix. Thus, by interpolating a cross-shape domain and an ellipse, we can obtain foliation of the bounds (1.3) and (1.4). This is the basic idea of our work. Based on this idea, we will show, by numerical computations, that each point inside the HS bounds is attained by cross-shaped domains whose sides are different (see section 3 for precise parameterizations of domains). We will also present a method to find a domain whose PT has a given pair of eigenvalues. We emphasize that the result of this paper is numerical and analytic computations of the PTs associated with the domains we construct in this work, for arbitrary aspect ratios, seem unlikely. However, we will show that it is possible to compute the eigenvalues of mixtures of ellipses and cross-shape domains in the high aspect ratio limit.

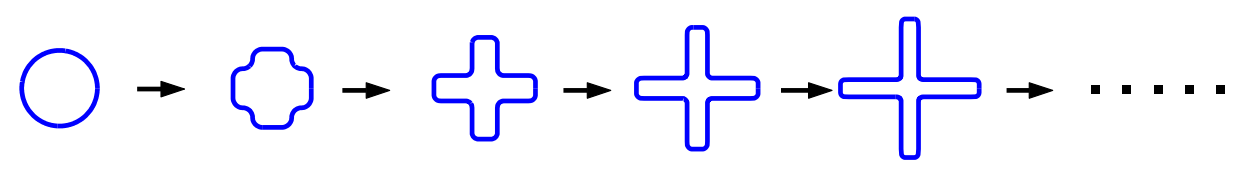

FiguRE 2. The variation of cross-domains starting from the unit disk.

The paper is organized as follows. In section 2, we review the definition of the $\mathrm{PT}$ and its representation in terms of boundary integrals. This boundary integral representation is used for our numerical computations throughout this paper. In sections 3 and 4, we parameterize the domains we consider using two parameters, one being the ellipticity of the ellipses (or the length over width ratio of the crosses' legs) and the second being the proportion of ellipses and crosses, to show numerical 
evidence of the fact that the corresponding PTs make foliation of the HS bounds. In section 5 we first provide the asymptotic behavior of the PT associated with crosses as crosses get thinner and longer. We then show that this results validates the numerical results presented in section 4 for thin an long inclusions - which are the most difficult to accurately simulate numerically.

In section 6 , we present a method to identify a parameterized domain whose PT has a given pair of eigenvalues. We derive an approximate formula to extrapolate from the computed eigenvalue pairs data the shape of the domain. We use a combination of least square fitting with Lagrange interpolation. The robustness of this extrapolation, compared to the direct simulation, is accurately tested.

In concluding this introduction, we briefly mention a conjecture of Pólya-Szegö. Observe that among the points in HS-bounds, the intersection point of the lower bound and the line $\lambda_{1}=\lambda_{2},(2(k-1) /(k+1), 2(k-1) /(k+1)$, has the minimal trace, and this is the eigenvalue of the PT associated with the disk of unit area. Pólya and Szegö [21] conjectured that the disk (or the sphere for three dimensions) is a unique domain with minimal trace. This conjecture has not yet been proved.

\section{Preliminaries}

Let us recall the definition of PT. Let $D$ be a bounded Lipschitz domain in $\mathbb{R}^{2}$. The conductivity of $\mathbb{R}^{2} \backslash \bar{D}$ and $D$ are assumed to be 1 and $0<k \neq 1<+\infty$, respectively. Let $W^{1,2}$ be the collection of all functions $\psi$ such that $\int_{\mathbb{R}^{2}}|\nabla \psi|^{2}<+\infty$ and $\psi(x)=O\left(|x|^{-1}\right)$. The polarization tensor $M=\left(M_{i j}\right)_{i, j=1,2}$ associated with the domain $D$ is defined to be the $2 \times 2$ matrix given by

$$
M_{i j}=(k-1)\left[\delta_{i j}|D|+\left.\int_{\partial D} x_{j} \frac{\partial \psi_{i}}{\partial \nu}\right|_{-} d s\right], \quad i, j=1,2,
$$

where $\psi_{i}, i=1,2$, is the unique solution in $W^{1,2}$ to the following transmission problem:

$$
\Delta \psi_{i}=(1-k) \nabla \cdot\left(\chi(D)\left(\nabla \psi_{i}+e_{i}\right)\right)
$$

or equivalently,

$$
\left\{\begin{array}{l}
\Delta \psi_{i}=0 \quad \text { in }\left(\mathbb{R}^{2} \backslash \bar{D}\right) \cup D \\
\left.\psi_{i}\right|_{+}-\left.\psi_{i}\right|_{-}=0 \quad \text { on } \partial D \\
\left.\frac{\partial \psi_{i}}{\partial \nu}\right|_{+}-\left.k \frac{\partial \psi_{i}}{\partial \nu}\right|_{-}=(k-1) \nu_{i} \quad \text { on } \partial D \\
\psi_{i}(x)=O\left(|x|^{-1}\right) \quad \text { as }|x| \rightarrow \infty
\end{array}\right.
$$

Here, $\chi(D)$ is the characteristic function of $D, e_{i}$ is the standard basis of $\mathbb{R}^{2}$, the subscripts + and - denote the limits from outside and inside $D$, respectively, and $\nu=\left(\nu_{1}, \nu_{2}\right)$ is the unit outward normal to $\partial D$. Then one can show (see [12]) that for any $\xi \in \mathbb{R}^{2}$

$$
M \xi \cdot \xi=\int_{\mathbb{R}^{2}} \gamma_{D}\left|\nabla w+\frac{k-1}{k} \chi(D) \xi\right|^{2}+\frac{k-1}{k}|\xi|^{2}|D|,
$$

where $w$ is the solution to

$$
\Delta w=(1-k) \nabla \cdot(\chi(D)(\nabla w+\xi)) .
$$


Here and throughout this paper $\gamma_{D}:=1+(k-1) \chi(D)$. Moreover, the solution $w$ is the minimizer of the functional

$$
\int_{\mathbb{R}^{2}} \gamma_{D}\left|\nabla \phi+\frac{k-1}{k} \chi(D) \xi\right|^{2}+\frac{k-1}{k}|\xi|^{2}|D|
$$

over $\phi \in W^{1,2}$. It then follows that

$$
\int_{\mathbb{R}^{n}} \gamma_{D}\left|\nabla w+\chi(D) \frac{k-1}{k} \xi\right|^{2}=-\int_{\mathbb{R}^{2}} \gamma_{D}|\nabla w|^{2}+\frac{(k-1)^{2}}{k}|\xi||D| .
$$

See [12] for details.

The definition (2.1) of PT is proved to be equivalent to the following representation using layer potential techniques $[2,1]$. For $\phi \in L^{2}(\partial D)$, let $\mathcal{K}_{D}^{*} \phi$ be defined by

$$
\mathcal{K}_{D}^{*} \phi(x)=\frac{1}{2 \pi} \text { p.v. } \int_{\partial D} \frac{\left\langle x-y, \nu_{x}\right\rangle}{|x-y|^{2}} \phi(y) d \sigma(y),
$$

where p.v. stands for the Cauchy principal value. Then $M$ can be represented as follows:

$$
M_{i j}=\int_{\partial D} y_{j}\left(\lambda I-\mathcal{K}_{D}^{*}\right)^{-1}\left(\nu_{i}\right)(y) d \sigma(y), \quad \lambda:=\frac{k+1}{2(k-1)} .
$$

This boundary integral representation of PT is particularly useful for computational purpose and we will use this representation for all the computations provided in this paper.

\section{Parameterizations of domains and attainability}

In this section, we show numerically that every point inside the bounds (1.3) and (1.4) is a pair of eigenvalues of a PT associated with a simply connected domain of unit area. The domains varies from ellipses to crosses as shown in the introduction. The crosses to be considered in this section will be slightly different from those considered in the previous section in order to avoid the corners.

Let us briefly explain how we numerically compute the PT $M$. The domain $D$ to be considered is symmetric with respect to $x$ and $y$ axes, and centered at the origin $(0,0)$. In order to compute $M$ using its definition given in (2.5), we compute $\left(\lambda I-\mathcal{K}_{D}^{*}\right)^{-1}\left(\nu_{i}\right)(y)$ in the following way. We first choose $2 m$ points $x_{1}, x_{2}, \ldots, x_{2 m}$ on $\partial D$, and find $\phi_{i}, i=1,2$, satisfying

$$
\left(\lambda I-\mathcal{K}_{D}^{*}\right) \phi_{i}\left(x_{k}\right)=\nu_{i}\left(x_{k}\right), \quad k=1,2, \cdots, 2 m .
$$

This equation is solved by making use of the collocation method and trapezoid rule (see, for instance, [18]). Because of the symmetry of $D$, the discretized operator $\left(\lambda I-\mathcal{K}_{D}^{*}\right)$ is a matrix of the form $\left(\begin{array}{cc}\mathcal{A} & \mathcal{B} \\ \mathcal{B} & \mathcal{A}\end{array}\right)$ where $\mathcal{A}$ and $\mathcal{B}$ are $m \times m$ matrices, and the vector $\nu_{i}=\nu_{i}\left(x_{k}\right)$ for $k=1, \ldots, 2 m$, takes the form $\left(\begin{array}{c}a_{i} \\ -a_{i}\end{array}\right)$. Therefore, the solution $\phi_{i}=\left(\phi_{i}\left(x_{k}\right)\right)_{k=1}^{2 m}$ should be of the form $\left(\begin{array}{c}b_{i} \\ -b_{i}\end{array}\right)$, where the vector $b_{i} \in \mathbb{R}^{m}$ is the solution of the algebraic equation

$$
(\mathcal{A}-\mathcal{B}) b_{i}=a_{i}, \quad i=1,2 .
$$

In order to solve (3.1), we use the decomposition $P(\mathcal{A}-\mathcal{B})=L U$ where the matrix $L, U$ and $P$ are a lower triangular, an upper triangular, and a permutation matrix, 
respectively. Once (3.1) is solved, we can in a standard way use (2.5) to finally calculate $M$.

As it will be shown in the following, the domain $D$ can be a thin long cross. As the cross gets thinner and longer, we need more points on $\partial D$, and hence the matrix $\mathcal{A}-\mathcal{B}$ becomes larger, causing more difficulties for solving (3.1). But, thanks to the symmetry of $D, \mathcal{A}-\mathcal{B}$ is a sparse matrix and then we can decompose it into several symmetric matrices of small sizes.

Note that, because of an obvious symmetry reason, it suffices to consider the region $\lambda_{2} \geq \lambda_{1}$. In fact, we recall that if two domains $D$ and $D^{\prime}$ are related by $D^{\prime}=R(D)$ where $R$ is a unitary transformation, then their corresponding PTs, $M^{\prime}$ and $M$, satisfy the relation:

$$
M^{\prime}=R M R^{T}
$$

For $t \geq 1$, let $E(t)$ be the ellipse of the form

$$
\frac{x^{2}}{a^{2}}+\frac{y^{2}}{b^{2}}=1, \quad a \leq b,
$$

where $t:=b / a$ and the area $|E(t)|=1$. The cross-shaped domain $D(t), t \geq 2$, is defined by

$$
D(t):=\{(x, y),(x,-y),(-x, y),(-x,-y) \mid(x, y) \in D\},
$$

where $|D(t)|=1$, and for $x \geq 0$ and $y \geq 0, t:=r_{1} / r_{2}$, and the set $D$ is given by

$$
D= \begin{cases}\left(r_{1}, y\right) & \text { for } 0 \leq y \leq \frac{r_{2}}{2}, \\ \left(r_{1}-\frac{r_{2}}{2}+\frac{r_{2}}{2} \frac{x}{\sqrt{x^{2}+y^{2}}}, \frac{r_{2}}{2}+\frac{r_{2}}{2} \frac{y}{\sqrt{x^{2}+y^{2}}}\right) & \text { for } 0 \leq \tan ^{-1} \frac{y}{x} \leq \frac{\pi}{2}, \\ \left(x, r_{2}\right) & \text { for } \frac{3 r_{2}}{2} \leq x \leq r_{1}-\frac{r_{2}}{2}, \\ \left(\frac{3 r_{2}}{2}+\frac{r_{2}}{2} \frac{x}{\sqrt{x^{2}+y^{2}}}, \frac{3 r_{2}}{2}+\frac{r_{2}}{2} \frac{y}{\sqrt{x^{2}+y^{2}}}\right) & \text { for } \pi \leq \tan ^{-1} \frac{y}{x} \leq \frac{3 \pi}{2}, \\ \left(r_{2}, y\right) & \text { for } \frac{3 r_{2}}{2} \leq y \leq r_{1}-\frac{r_{2}}{2}, \\ \left(\frac{r_{2}}{2}+\frac{r_{2}}{2} \frac{x}{\sqrt{x^{2}+y^{2}}}, r_{1}-\frac{r_{2}}{2}+\frac{r_{2}}{2} \frac{y}{\sqrt{x^{2}+y^{2}}}\right) & \text { for } 0 \leq \tan ^{-1} \frac{y}{x} \leq \frac{\pi}{2}, \\ \left(x, r_{1}\right) & \text { for } 0 \leq x \leq \frac{r_{2}}{2} .\end{cases}
$$

The domain $D(t)$ is a cross whose corners are polished to be arcs. We make the corners smooth to make the computations easier. Figure 3 shows the shape of $D(t=3)$. The classes of domains $E(t)$ and $D(t)$ are the basic building blocks for representing the optimal shapes constructed in this paper.

It is known that the eigenvalue pair of the PT of the ellipse $E(t)$ lies on the lower bound (hyperbola) of the region $A$. In fact, according to [8], the PT for the ellipse $E(t)$ is given by

$$
M=|E|\left(\begin{array}{cc}
\frac{(k-1)(a+b)}{a+k b} & 0 \\
0 & \frac{(k-1)(a+b)}{k a+b}
\end{array}\right) .
$$

Thus the eigenvalue pair of the PT for $E(t)$ is

$$
\left(\frac{(k-1)(1+t)}{1+k t}, \frac{(k-1)(1+t)}{k+t}\right) .
$$




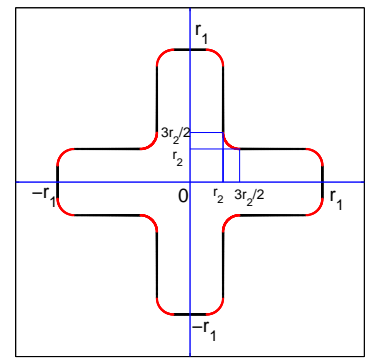

Figure 3. The graph of $D(t)$ for $t=r_{1} / r_{2}=3$.
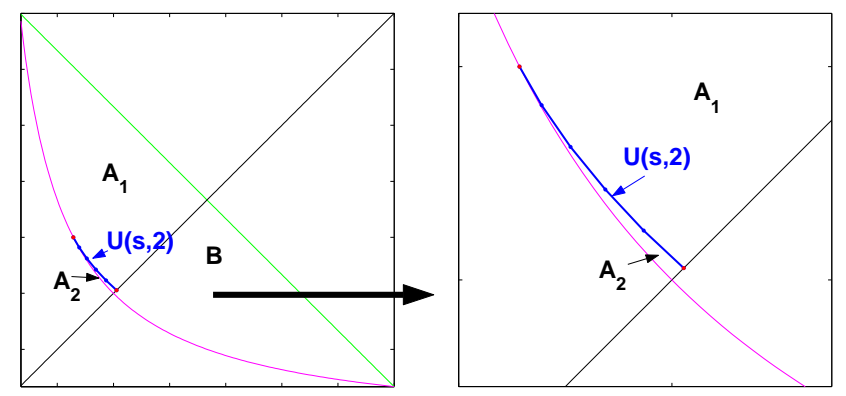

Figure 4. The curve of PT associated with $U(s, 2)$ divides $A$ into the upper part $A_{1}$ and the lower part $A_{2}$.

On the other hand, since the domain $D(t)$ is invariant under the rotation by $\pi / 2$, by the relation (3.2) the eigenvalue pair of its PT lies on the line $\lambda_{1}=\lambda_{2}$.

Let

$$
U(s, 2)=(1-s) D(2)+s E(2), \quad 0 \leq s \leq 1 .
$$

As shown in Figure 4, the eigenvalue pair of the PT associated with the domain $U(s, 2)$ divides the region $A$ into two parts $A_{1}$ and $A_{2}$. Therefore, we will use different parameterizations of domains to attain points in $A_{1}$ and $A_{2}$. This is solely for the convenience of the computations.

\section{Attainability of the region $A_{1}$. Let}

$$
U(s, t)=(1-s) D(t)+s E(t), \quad 0 \leq s \leq 1, \quad 2 \leq t .
$$

By this notation, we do not mean the convex combination of the two shapes. Notice for every $t$, that $D(t)$ and $E(t)$ are both star-shaped domains around the origin, i.e., there exist two functions $d_{t} \in C^{1}([0,2 \pi])$ and $e_{t} \in C^{\infty}([0,2 \pi])$ such that $\partial D(t)=\left\{\left(\varphi, d_{t}(\varphi)\right), \varphi \in[0,2 \pi]\right\}$ and $\partial E(t)=\left\{\left(\varphi, e_{t}(\varphi)\right), \varphi \in[0,2 \pi]\right\}$. The precise definition of $U(s, t)$ for a given $s \in[0,1]$ is the bounded domain limited by the $C^{1}$ curve $\partial U(s, t)=\left\{\left(\varphi,(1-s) d_{t}(\varphi)+s e_{t}(\varphi)\right), \varphi \in[0,2 \pi]\right\}$. Note that $|U(s, t)|=$ $\frac{1}{2} \int_{0}^{2 \pi}\left((1-s) d_{t}(\varphi)+s e_{t}(\varphi)\right)^{2} d \varphi$ is not a priori equal to one. We therefore rescale the polarization tensor by a factor $1 /|U(s, t)|$.

Our goal in this section is to show that the points inside $A_{1}$ are attained by the domain $U(s, t)$. Note that $U(1, t)=E(t)$ is an ellipse and $U(0, t)=D(t)$ is 
a cross. As $t$ gets larger, the ellipse $U(1, t)$ becomes thinner and the cross $U(0, t)$ becomes thinner and longer. For a fixed $t, U(s, t)$ changes its shape from a cross to an ellipse as $s$ varies from 0 to 1 . The left-hand side of Figure 5 shows the variation of the domain $U(s, t)$. Numerical computations show that as $t \rightarrow \infty$, the eigenvalue pair of PT associated with $U(0, t)$ moves toward the point $\left(\lambda_{1}, \lambda_{2}\right)=$ $((k-1)(k+1) /(2 k),(k-1)(k+1) /(2 k))$ following the line $\lambda_{1}=\lambda_{2}$ while that of $U(1, t)$ moves toward the point $((k-1) / k, k-1)$ following the hyperbola. Both of these facts will be rigorously established in section 5 . For each fixed $t$, the eigenvalue pair for $U(s, t), 0 \leq s \leq 1$, makes a curve in the region $A_{1}$, and these curves make foliation of the region $A_{1}$ as $t$ varies. Figure 5 shows variations of $U(s, t)$ and the result of numerical computations.
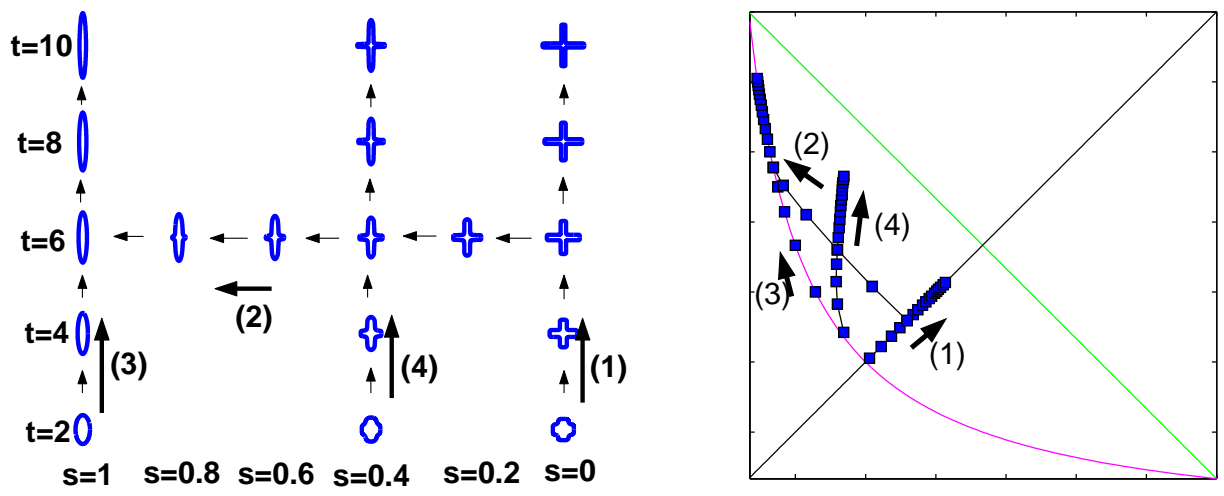

Figure 5. The variation of $U(s, t)$ and the corresponding eigenvalue pairs. As $t \rightarrow \infty$, the cross and ellipse become thinner and longer, and corresponding eigenvalues move toward the upper bound. As $s \rightarrow 1$, the cross becomes an ellipse and corresponding eigenvalues move toward the lower hyperbola.

\section{Attainability of the region $A_{2}$. Let}

$$
L(s, t):=(1-s) E(t)+s D(2), \quad 0 \leq s \leq 1 \text { and } 1 \leq t<2 .
$$

As above, this notation does not mean the convex combination of $E(t)$ and $D(2)$ but the bounded domain limited by the curve $\left\{\left(\varphi,(1-s) e_{t}(\varphi)+s d_{2}(\varphi)\right), \varphi \in[0,2 \pi]\right\}$. Note that $D(2)$ is fixed. For each fixed $t, E(t)$ corresponds to a point on the lower hyperbola. So, $L(s, t)$ interpolates the point on the hyperbola and the fixed point on the line $\lambda_{1}=\lambda_{2}$ corresponding to $D(2)$. Numerical computations give the evidence of the fact that the points in $A_{2}$ arise as the eigenvalue pairs of the PT corresponding to the domain $L(s, t)$. Figure 6 shows the variation of $L(s, t)$ and the corresponding eigenvalues of its PT.

Figure 7 shows attainability of both the regions $A_{1}$ and $A_{2}$.

\section{An APPROXimate FORMUla FOR the FOLIATION}

In the previous section, we parameterized domains to show that as the parameter $s$ varies from 0 to 1 for a fixed $t$, the eigenvalue pairs make a curve. Then, by varying $t$ these curves, a foliation of the region, say $A_{1}$, can be obtained. 

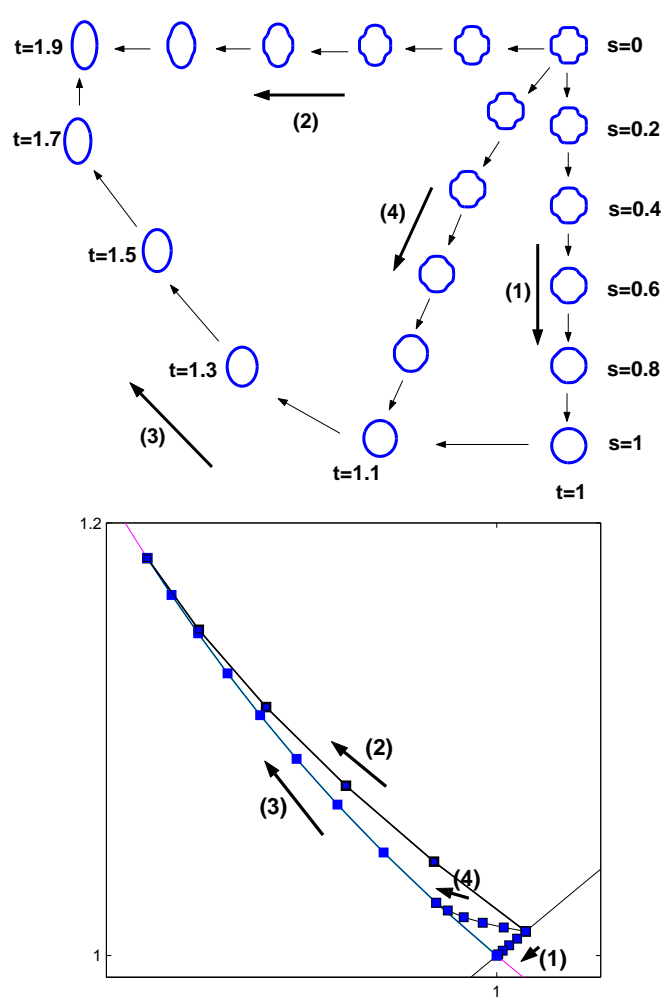

FIgURE 6 . The variations of $L(s, t)$ and the corresponding eigenvalue pairs of its $\mathrm{PT}$.

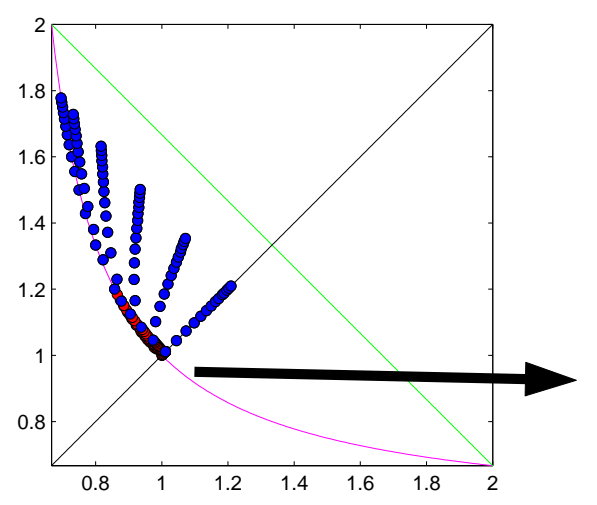

(a)

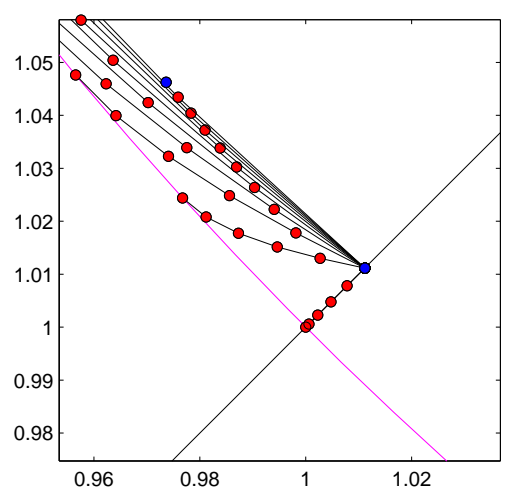

(b)

Figure 7. The left figure shows the eigenvalue pairs of the PTs associated with $U(s, t)$ and $L(s, t)$. The blue points are eigenvalue pairs corresponding to $U(s, t)$ and the red ones to $L(s, t)$. The right figure enlarges $A_{2}$. 
In this section, we derive an approximate formula for this foliation. Since the formula for the region $A_{2}$ can be obtained in almost the same way, we omit its derivation.

Choose finite sets of parameters, $S=\left\{s_{0}=0, s_{1}, \ldots, s_{m}=1\right\}$ and $T=$ $\left\{t_{0}=2, t_{1}, \ldots, t_{n}\right\}$. Then numbers $m, n$ are at our disposal. We may take large ones if we need very accurate approximations. We then compute the eigenvalues $\left(\lambda_{1}\left(s_{i}, t_{j}\right), \lambda_{2}\left(s_{i}, t_{j}\right)\right)$ of the PT associated with the domain $U\left(s_{i}, t_{j}\right)$ for $i=0, \ldots, m$ and $j=0, \ldots, n$. Our objective is to find a formula to relate $\lambda_{1}\left(s_{i}, t_{j}\right)$ with $\lambda_{2}\left(s_{i}, t_{j}\right)$.

Let us introduce a transformation which makes the computations easier. Let $V$ be given by

$$
V(x, y):=R_{\frac{\pi}{4}}\left(x-\frac{(k-1)(k+1)}{2 k}, y-\frac{(k-1)(k+1)}{2 k}\right),
$$

where $R_{\theta}$ is the rotation by $\theta$. Then define

$$
\left(\rho_{1}(s, t), \rho_{2}(s, t)\right):=V\left(\lambda_{1}(s, t), \lambda_{2}(s, t)\right) .
$$

We seek to find a function $P(x, t)$ of the form

$$
P(x, t)=\sum_{l=0}^{m} a_{l}(t) x^{l}
$$

such that

$$
\rho_{2}\left(s_{i}, t_{j}\right)=P\left(\rho_{1}\left(s_{i}, t_{j}\right), t_{j}\right)=\sum_{l=0}^{m} a_{l}\left(t_{j}\right) \rho_{1}\left(s_{i}, t_{j}\right)^{l} .
$$

We can obtain the desired formula by completing the following steps.

\section{An approximate formula for the foliation}

S1. For each $s_{i} \in S(i \neq m)$ and $l=1,2$, we look for a function $P_{i}^{l}$ on $(1,3 / 2]$ satisfying

$$
P_{i}^{l}\left(\frac{1+t_{j}}{t_{j}}\right)=\rho_{k}\left(s_{i}, t_{j}\right), \quad t_{j} \in T
$$

or minimizing

$$
\sum_{t_{j} \in T}\left|P_{i}^{l}\left(\frac{1+t_{j}}{t_{j}}\right)-\rho_{k}\left(s_{i}, t_{j}\right)\right|
$$

We obtain such a polynomial $P_{i}^{l}$ using the least square curve fitting.

If $i=m$ or $s_{i}=1$, then $U\left(s_{m}, t\right)$ is an ellipse, and the corresponding eigenvalues are given explicitly by (3.3). We use these explicit formulas for our $P_{m}^{l}, l=1,2$.

S2. We seek a function $P(x, t)$, which is the unique $m$-th degree polynomial in $x$ satisfying

$$
P\left(\rho_{1}\left(s_{i}, t_{j}\right), t_{j}\right)=\rho_{2}\left(s_{i}, t_{j}\right), \quad s_{i} \in S \text { and } t_{j} \in T
$$




\begin{tabular}{|c||c|c|c|c|c|}
\hline $\mathrm{t} \backslash \mathrm{s}$ & 0 & 0.2 & 0.4 & 0.8 & 1 \\
\hline \hline & & & & & \\
2 & $(1.0111,1.0111)$ & $(0.9736,1.0463)$ & $(0.9376,1.0847)$ & $(0.8778,1.1640)$ & $(0.8571,1.2000)$ \\
3 & $(1.0443,1.0443)$ & $(0.9812,1.1021)$ & $(0.9194,1.1656)$ & $(0.8227,1.2886)$ & $(0.8000,1.3333)$ \\
4 & $(1.0737,1.0737)$ & $(0.9945,1.1480)$ & $(0.9160,1.2292)$ & $(0.7938,1.3803)$ & $(0.7692,1.4286)$ \\
5 & $(1.0981,1.0981)$ & $(1.0074,1.1852)$ & $(0.9171,1.2798)$ & $(0.7767,1.4498)$ & $(0.7500,1.5000)$ \\
6 & $(1.1182,1.1182)$ & $(1.0187,1.2158)$ & $(0.9195,1.3208)$ & $(0.7656,1.5042)$ & $(0.7368,1.5556)$ \\
7 & $(1.1351,1.1351)$ & $(1.0285,1.2413)$ & $(0.9220,1.3548)$ & $(0.7578,1.5482)$ & $(0.7273,1.6000)$ \\
8 & $(1.1491,1.1491)$ & $(1.0366,1.2627)$ & $(0.9243,1.3833)$ & $(0.7520,1.5844)$ & $(0.7200,1.6364)$ \\
9 & $(1.1622,1.1622)$ & $(1.0443,1.2815)$ & $(0.9268,1.4078)$ & $(0.7475,1.6147)$ & $(0.7143,1.6667)$ \\
10 & $(1.1717,1.1717)$ & $(1.0497,1.2970)$ & $(0.9282,1.4286)$ & $(0.7438,1.6407)$ & $(0.7097,1.6923)$ \\
11 & $(1.1811,1.1811)$ & $(1.0552,1.3111)$ & $(0.9299,1.4470)$ & $(0.7410,1.6629)$ & $(0.7059,1.7143)$ \\
12 & $(1.1881,1.1881)$ & $(1.0591,1.3229)$ & $(0.9308,1.4629)$ & $(0.7382,1.6824)$ & $(0.7027,1.7333)$ \\
13 & $(1.1964,1.1964)$ & $(1.0640,1.3344)$ & $(0.9325,1.4773)$ & $(0.7361,1.6995)$ & $(0.7000,1.7500)$ \\
14 & $(1.2025,1.2025)$ & $(1.0675,1.3441)$ & $(0.9335,1.4901)$ & $(0.7342,1.7147)$ & $(0.6977,1.7647)$ \\
15 & $(1.2077,1.2077)$ & $(1.0703,1.3527)$ & $(0.9341,1.5013)$ & $(0.7325,1.7281)$ & $(0.6957,1.7778)$ \\
16 & $(1.2134,1.2134)$ & $(1.0736,1.3609)$ & $(0.9351,1.5117)$ & $(0.7309,1.7403)$ & $(0.6939,1.7895)$ \\
17 & $(1.2205,1.2205)$ & $(1.0779,1.3690)$ & $(0.9368,1.5213)$ & $(0.7299,1.7511)$ & $(0.6923,1.8000)$ \\
18 & $(1.2272,1.2272)$ & $(1.0819,1.3764)$ & $(0.9384,1.5301)$ & $(0.7282,1.7613)$ & $(0.6909,1.8095)$ \\
& & & & & \\
\hline
\end{tabular}

TABLE 1 . The eigenvalue pairs $\left(\lambda_{1}^{U}(s, t), \lambda_{2}^{U}(s, t)\right)$ associated with $U(s, t)$.

Such a polynomial is given by

$$
\begin{aligned}
P(x, t) & =\sum_{i=0}^{m} a_{i}(t) x^{i} \\
& =\sum_{i=0}^{m} \prod_{\substack{l \neq i \\
l=0, \ldots, m}}\left(\rho_{2}\left(s_{i}, t\right) \frac{x-\rho_{1}\left(s_{l}, t\right)}{\rho_{1}\left(s_{i}, t\right)-\rho_{1}\left(s_{l}, t\right)}\right) \\
& =\sum_{i=0}^{m} \prod_{\substack{l \neq i \\
l=0, \ldots, m}}\left(P_{i}^{2}\left(\frac{1+t}{t}\right) \frac{x-P_{l}^{1}\left(\frac{1+t}{t}\right)}{P_{i}^{1}\left(\frac{1+t}{t}\right)-P_{l}^{1}\left(\frac{1+t}{t}\right)}\right),
\end{aligned}
$$

where $P_{i}^{1}, P_{i}^{2}, i=0, \ldots, m$, are the polynomials obtained in step S1.

Notice that for the sake of a simpler parameterization we have used two different ways of interpolation for the lower and upper parts.

Figure 8 shows results of numerical tests using Matlab program. For these computations we assume the conductivity $k=3$. The boundary integral is discretized at 16,000 boundary points. This large number of points will be used throughout the paper. After doing experiments on the accuracy of the method, we have chosen this number to make the numerical computations precise, since as the cross gets thinner, the domain variations affect very little the PT. The dots in the left figure of Figure 8 are the computed eigenvalue pairs of the PT associated with $U(s, t)$ for $s=0,0.2, \cdots, 1$ and $t=2,3, \cdots, 18$. The solid-curves are graphs of $P(x, t)$ fitting dots, which are computed following the procedure described in steps S1 and S2. We use $S:=\{0,0.4,1\}$ and $T:=\{2,3, \cdots, 18\}$ for the computation of $P(x, t)$. The right hand side figure is for $L(s, t), s=1,1.1, \ldots, 1.9, t=2,3, \cdots, 18$. Numerical values for the eigenvalues are recorded in Tables 1 and 2. Figure 9 shows whole foliation by $P(x, t)$ of the HS bounds. 

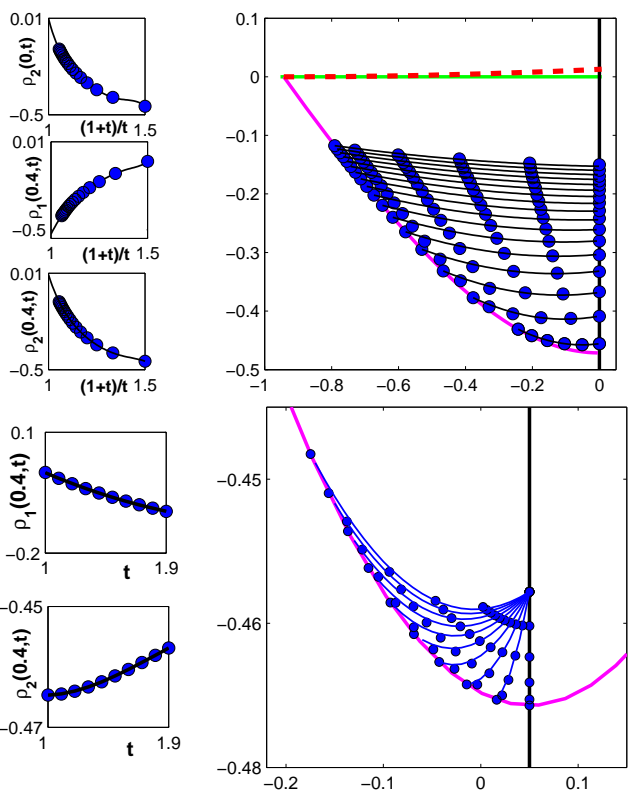

FIgURE 8. Numerical results for $U$ and $L$. The dots are the computed eigenvalue pairs of the PT of the domains and solid curves are curves fitting dots.

\begin{tabular}{|c||c|c|c|c|c|}
\hline $\mathrm{t} \backslash \mathrm{s}$ & 0 & 0.2 & 0.4 & 0.8 & 1 \\
\hline \hline & & & & & \\
1 & $(1.0111,1.0111)$ & $(1.0078,1.0078)$ & $(1.0048,1.0048)$ & $(1.0006,1.0006)$ & $(1.0000,1.0000)$ \\
1.1 & $(1.0111,1.0111)$ & $(1.0027,1.0130)$ & $(0.9946,1.0152)$ & $(0.9812,1.0208)$ & $(0.9767,1.0244)$ \\
1.2 & $(1.0111,1.0111)$, & $(0.9981,1.0178)$ & $(0.9856,1.0248)$ & $(0.9642,1.0399)$ & $(0.9565,1.0476)$ \\
1.3 & $(1.0111,1.0111)$ & $(0.9940,1.0222)$ & $(0.9775,1.0339)$ & $(0.9491,1.0581)$ & $(0.9388,1.0698)$ \\
1.4 & $(1.0111,1.0111)$ & $(0.9903,1.0264)$ & $(0.9702,1.0424)$ & $(0.9357,1.0753)$ & $(0.9231,1.0909)$ \\
1.5 & $(1.0111,1.0111)$ & $(0.9869,1.0302)$ & $(0.9636,1.0504)$ & $(0.9237,1.0917)$ & $(0.9091,1.1111)$ \\
1.6 & $(1.0111,1.0111)$ & $(0.9838,1.0338)$ & $(0.9575,1.0580)$ & $(0.9128,1.1074)$ & $(0.8966,1.1304)$ \\
1.7 & $(1.0111,1.0111)$ & $(0.9810,1.0372)$ & $(0.9520,1.0652)$ & $(0.9029,1.1224)$ & $(0.8852,1.1489)$ \\
1.8 & $(1.0111,1.0111)$ & $(0.9783,1.0404)$ & $(0.9468,1.0720)$ & $(0.8938,1.1368)$ & $(0.8750,1.1667)$ \\
1.9 & $(1.0111,1.0111)$ & $(0.9759,1.0434)$ & $(0.9421,1.0785)$ & $(0.8855,1.1506)$ & $(0.8657,1.1837)$ \\
& & & & & \\
\hline
\end{tabular}

TABLE 2. The eigenvalue pairs $\left(\lambda_{1}^{L}(s, t), \lambda_{2}^{L}(s, t)\right)$ associated with $L(s, t)$.

\section{Asymptotics of PT For thin Crosses, And Analytic acCuracy of the FOLIATION}

On Figure 5 we see that the upper trace bound is reached by the PT corresponding to $E(t)$ as $t$ tends to infinity. It also seems that as the cross becomes thinner and longer, the corresponding PT approaches the upper trace bound.

The first result of this section show that this is indeed what is predicted by the asymptotics. 


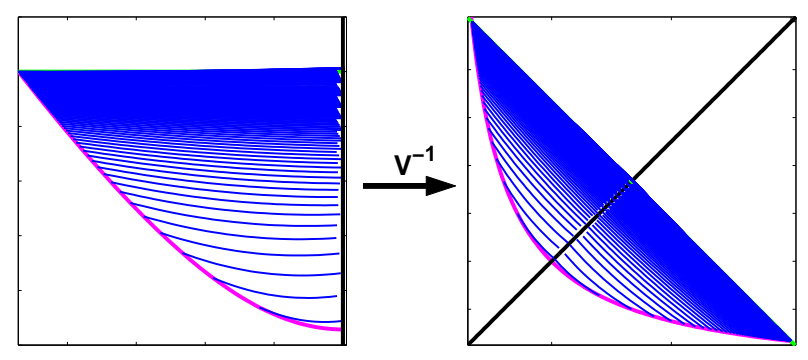

Figure 9. The numerical results for the foliation.

Theorem 5.1. The PT $M_{D(t)}$ associated to $D(t)$ satisfies

$$
\lim _{t \rightarrow \infty} M_{D(t)}=\frac{(k-1)(k+1)}{2 k}\left(\begin{array}{ll}
1 & 0 \\
0 & 1
\end{array}\right) .
$$

On Figure 9, it seems that as the "thinness" parameter $t$ becomes large, the curve described by $\left(\lambda_{1}^{U}(s, t), \lambda_{2}^{U}(s, t)\right)$ as $s$ varies between 0 and 1 appears to be a straight line. This can be measured on table 1 as well. The cross-shape was designed to be "simple" from a computational point of view. The drawback is that obtaining precise analytical results for this shape is unreasonably technical. We shall show that the affine nature of the curve $\left(\lambda_{1}^{U}(s, t), \lambda_{2}^{U}(s, t)\right)$ is asymptotically correct for a similar (but different) cross for which analytical computations are simpler.

The alternate cross we consider is given as follows. Let $F_{t}$ and $S_{t}$ be a "flat" elliptic beam and a "standing" elliptic beam given by

$$
F_{t}: \frac{x^{2}}{b^{2}}+\frac{y^{2}}{a^{2}}=1, S_{t}: \frac{x^{2}}{a^{2}}+\frac{y^{2}}{b^{2}}=1, a=\frac{1}{\sqrt{2 \pi t}}, b=t a .
$$

Define $X_{t}:=F_{t} \cup S_{t}$, and following the approach introduced in section 3 , define for all $s \in[0,1]$ and $t \in[1, \infty)$,

$$
U(s, t)=s E(t)+(1-s) X(t)
$$

the domain of unit area obtained by radial interpolation between $X(t)$ and $E(t)$. The second result of this section shows that as $s$ varies between 0 and 1 , the curve described by $\left(\lambda_{1}^{U}(s, t), \lambda_{2}^{U}(s, t)\right)$ is asymptotically a line.

Theorem 5.2. The PT $M_{U(s, t)}$ associated to $U(s, t)$ satisfies

$M_{U(s, t)}=\frac{(\sqrt{2} s+1-s)^{2}}{(\sqrt{2} s+1-s)^{2}+(1-s)^{2}} M_{U(1, t)}+\frac{(1-s)^{2}}{(\sqrt{2} s+1-s)^{2}+(1-s)^{2}} M_{U(0, t)}+\epsilon(t)$,

where $\epsilon(t)$ tends to zero as $t$ tends to infinity.

We shall start by proving Theorem 5.1.

Note that $D(t)$ can be approximated by $\tilde{D}_{t}=H_{t} \cup V_{t}$, where

$$
H_{t}=\left[-\sqrt{\frac{t}{8}}, \sqrt{\frac{t}{8}}\right] \times\left[-\frac{1}{\sqrt{8 t}}, \frac{1}{\sqrt{8 t}}\right] \text { and } V_{t}=\left[-\frac{1}{\sqrt{8 t}}, \frac{1}{\sqrt{8 t}}\right] \times\left[-\sqrt{\frac{t}{8}}, \sqrt{\frac{t}{8}}\right] \text {. }
$$

The difference between the two domains are the smooth corners of $D(t)$, and the surface of $H_{t} \cup V_{t}$ which is not exactly one. It is easy to see that, for every $t>0$ 
there exists $t_{1}$ and $t_{2}$ and $\lambda$ such that

$$
\left|t_{1}-t_{2}\right|+|\lambda-1| \leq \frac{C}{t}
$$

where $C$ is a constant independent of $t$, and $\tilde{D}_{t_{1}} \subset D(t) \subset \lambda \tilde{D}_{t_{2}}$. Since the polarization tensors are monotonous with respect to inclusion, we deduce that

$$
M_{D(t)}=M_{\tilde{D}(t)}+O\left(\frac{1}{t}\right)
$$

In the sequel, we will identify $D$ and $\tilde{D}(t)$. The asymptotic limit of the polarization tensor of a single thin beam is known [7]. We have

$$
\lim _{t \rightarrow \infty} M_{H(t)}=\frac{1}{2}\left(\begin{array}{cc}
k-1 & 0 \\
0 & \frac{k-1}{k}
\end{array}\right) \text {, and } \lim _{t \rightarrow \infty} M_{V(t)}=\frac{1}{2}\left(\begin{array}{cc}
\frac{k-1}{k} & 0 \\
0 & k-1
\end{array}\right) .
$$

Given $\xi \in \mathbb{R}^{2}$, let $w_{t}^{\xi} \in W^{1,2}$ be the solution to

$$
\Delta w_{t}=(1-k) \nabla \cdot\left(\chi(D(t))\left(\nabla w_{t}+\xi\right)\right)
$$

and let $h_{t}^{\xi}$, and $v_{t}^{\xi}$ be the solutions to the same equations with $D(t)$ replaced with $H_{t}$ and $V_{t}$ respectively.

The following lemmas hold.

Lemma 5.3. For any unit vector $\xi=\left(\xi_{1}, \xi_{2}\right)$, the functions $h_{t}^{\xi}$ and $v_{t}^{\xi}$ satisfy $(5.2)$

$\left\|\nabla h_{t}^{\xi}+\chi\left(H_{t}\right) \frac{1-k}{k} \xi_{2} e_{2}\right\|_{L^{2}\left(\mathbb{R}^{2}\right)}=\epsilon(t)$, and $\left\|\nabla v_{t}^{\xi}+\chi\left(V_{t}\right) \frac{1-k}{k} \xi_{1} e_{1}\right\|_{L^{2}\left(\mathbb{R}^{2}\right)}=\epsilon(t)$,

where $\lim _{t \rightarrow \infty} \epsilon(t)=0$.

Proof. Write $M\left(H_{t}\right)=\left(M_{t}^{i, j}\right)_{i, j=1,2}$. Since

$$
M_{t}^{1,1}=\frac{k-1}{2}+\epsilon(t) \quad \text { as } t \rightarrow \infty,
$$

it follows from (2.3) and (2.4) that

$$
\int_{\mathbb{R}^{2}} \gamma_{H_{t}}\left|\nabla h_{t}^{e_{1}}\right|^{2}=\epsilon(t)
$$

On the other hand, since $M_{t}^{2,2}=(k-1) / 2 k+\epsilon(t)$ as $t \rightarrow \infty$, we get from (2.3) that

$$
\int_{\mathbb{R}^{2}} \gamma_{H_{t}}\left|\nabla h_{t}^{e_{2}}+\frac{k-1}{k} \chi\left(H_{t}\right) e_{2}\right|^{2}=\epsilon(t)
$$

Since $h_{t}^{\xi}=\xi_{1} h_{t}^{e_{1}}+\xi_{2} h_{t}^{e_{2}}$, we obtain the first limit in (5.2). The second estimate can be proved in a similar fashion.

Lemma 5.4. For each unit vector $\xi$, we have

$$
\left\|\nabla w_{t}^{\xi}-\left(\nabla h_{t}^{\xi}+\nabla v_{t}^{\xi}\right)\right\|_{L^{2}\left(\mathbb{R}^{2}\right)}=\epsilon(t)
$$

where $\lim _{t \rightarrow \infty} \epsilon(t)=0$. 
Proof. Let us, for simplicity, drop superscripts $\xi$. Since

$$
\left\|\nabla h_{t}\right\|_{L^{2}\left(H_{t} \cap V_{t}\right)} \leq\left\|\nabla h_{t}+\chi\left(H_{t}\right) \frac{1-k}{k} \xi_{2} e_{2}\right\|_{L^{2}\left(H_{t} \cap V_{t}\right)}+\xi_{2}\left|H_{t} \cap V_{t}\right|=\epsilon(t),
$$

it follows from (5.2) that

$$
\left\|\nabla h_{t}\right\|_{L^{2}\left(V_{t}\right)}=\epsilon(t) .
$$

Likewise we get

$$
\left\|\nabla v_{t}\right\|_{L^{2}\left(H_{t}\right)}=\epsilon(t) .
$$

By simple algebra, we obtain

$\nabla \cdot \gamma_{D(t)} \nabla\left(w_{t}-h_{t}-v_{t}\right)$

$$
=(k-1) \nabla \cdot\left(\chi\left(V_{t}\right) \nabla h_{t}+\chi\left(H_{t}\right) \nabla v_{t}\right)-(k-1) \nabla \cdot\left(\chi\left(H_{t} \cap V_{t}\right)\left(\nabla h_{t}+\nabla v_{t}+\xi\right)\right) .
$$

If we multiply the above equation by a test function $\phi$ and integrate by parts, we get

$$
\int_{\mathbb{R}^{2}} \gamma_{X_{t}} \nabla\left(w_{t}-h_{t}-v_{t}\right) \cdot \nabla \phi=(k-1)(A+B+C)
$$

with

$$
\begin{aligned}
|A| & =\left|\int_{H_{t} \cap V_{t}}\left(\nabla h_{t}+\nabla v_{t}+\xi\right) \cdot \nabla \phi\right| \leq\left\|\nabla h_{t}+\nabla v_{t}+\xi\right\|_{L^{2}\left(H_{t} \cap V_{t}\right)}\|\nabla \phi\|_{L^{2}\left(\mathbb{R}^{2}\right)}, \\
|B| & =\left|\int_{V_{t}} \nabla h_{t} \cdot \nabla \phi\right| \leq\left\|\nabla h_{t}\right\|_{L^{2}\left(V_{t}\right)}\|\nabla \phi\|_{L^{2}\left(\mathbb{R}^{2}\right)}, \\
|C| & =\left|\int_{H_{t}} \nabla v_{t} \cdot \nabla \phi\right| \leq\left\|\nabla v_{t}\right\|_{L^{2}\left(H_{t}\right)}\|\nabla \phi\|_{L^{2}\left(\mathbb{R}^{2}\right)} .
\end{aligned}
$$

Thanks to (5.4) and (5.5), we obtain

$$
\left|\int_{\mathbb{R}^{2}} \gamma_{D(t)} \nabla\left(w_{t}-h_{t}-v_{t}\right) \cdot \nabla \phi\right| \leq\|\nabla \phi\|_{L^{2}\left(\mathbb{R}^{2}\right)} \epsilon(t),
$$

for any test function $\phi$, and hence (5.3) follows.

We are now ready to prove Theorem 5.1.

Proof of Theorem 5.1. By (2.1) we have

$$
M_{D(t)} \xi \cdot \xi=(k-1)\left[1+(k-1) \int_{D(t)} \xi \cdot \nabla w_{t}^{\xi} d x\right]
$$

for any $\xi \in \mathbb{R}^{2}$. It then follows from (5.3) that

$$
M_{D(t)} \xi \cdot \xi=(k-1)\left[1+(k-1) \int_{D(t)} \xi \cdot\left(\nabla h_{a}^{\xi}+\nabla v_{d}^{\xi}\right) d x\right]+\epsilon(t) .
$$

From (5.2) we obtain that

$$
\begin{aligned}
\int_{D(t)} \xi \cdot\left(\nabla h_{t}^{\xi}+\nabla v_{t}^{\xi}\right) d x & =\int_{H_{t}} \xi \cdot \nabla h_{t}^{\xi}+\int_{V_{t}} \xi \cdot \nabla v_{t}^{\xi}+\int_{D(t) \backslash H_{t}} \xi \cdot \nabla h_{t}^{\xi}+\int_{D(t) \backslash V_{t}} \xi \cdot \nabla v_{t}^{\xi} \\
& =\int_{H_{t}} \xi \cdot \nabla h_{t}^{\xi}+\int_{V_{t}} \xi \cdot \nabla v_{t}^{\xi}+\epsilon(t) .
\end{aligned}
$$


Therefore, (5.6) yields

$$
M_{D(t)} \xi \cdot \xi=M_{V(t)} \xi \cdot \xi+M_{H(t)} \xi \cdot \xi+\epsilon(t) .
$$

Now, (5.1) immediately follows from (5).

Proof of Theorem 5.2. We have introduced

$$
U(s, t)=s E(t)+(1-s) X(t),
$$

that is, a domain with vertical and horizontal symmetries defined in the first quadrant as the domain inside the curve given, in polar coordinates, by

$$
\begin{aligned}
\text { for } 0 \leq \varphi \leq \frac{\pi}{4}, \rho_{U}(\varphi) & =\sqrt{\frac{t}{2 \pi}} *\left(\frac{\sqrt{2} s}{\sqrt{t^{2} \cos ^{2}(\varphi)+\sin ^{2}(\varphi)}}+\frac{1-s}{\sqrt{t^{2} \sin ^{2}(\varphi)+\cos ^{2}(\varphi)}}\right) \\
\text { for } \frac{\pi}{4} \leq \varphi \leq \frac{\pi}{2}, \rho_{U}(\varphi) & =\sqrt{\frac{t}{2 \pi}} * \frac{\sqrt{2} s+1-s}{\sqrt{t^{2} \cos ^{2}(\varphi)+\sin ^{2}(\varphi)}} .
\end{aligned}
$$

With this definition, we can compute

$$
|U(s, t)|=\int_{0}^{\frac{\pi}{4}} \frac{1}{2} \rho_{U}^{2}(\varphi) d \varphi+\int_{\frac{\pi}{4}}^{\frac{\pi}{2}} \frac{1}{2} \rho_{U}^{2}(\varphi) d \varphi=\frac{(1-s)^{2}}{2}+\frac{(\sqrt{2} s+1-s)^{2}}{2}+\epsilon(t) .
$$

Furthermore, we see that, up to a small volume, $U(s, t)=\tilde{F}_{s t} \cup \tilde{S}_{s t}+\epsilon(t)$, where

$$
\tilde{F}_{s t}: \frac{x^{2}}{\tilde{a}^{2}}+\frac{y^{2}}{\tilde{b}^{2}}=1, \tilde{S}_{s t}: \frac{x^{2}}{\tilde{c}^{2}}+\frac{y^{2}}{\tilde{d}^{2}}=1, a=\frac{\sqrt{2} s+1-s}{\sqrt{2 \pi t}}, b=t a
$$

and

$$
c=t d, d=\frac{(1-s)}{\sqrt{2 \pi t}} .
$$

Arguing as before, we see that the PT corresponding to $U(s, t)$ is equal to the PT corresponding to $\tilde{F}_{s t} \cup \tilde{S}_{s t}$, up to an error $\epsilon(t)$ which tends to zero as $t$ tends to infinity. The polarization tensors of $\tilde{F}_{s t}$ and $\tilde{S}_{s t}$ are known [8] and given by (3.3). Thus we have

$$
\lim _{t \rightarrow \infty} M_{F_{s t}}=\left\|F_{s t}\right\|\left(\begin{array}{cc}
k-1 & 0 \\
0 & \frac{k-1}{k}
\end{array}\right) \text {, and } \lim _{t \rightarrow \infty} M_{S(t)}=\left\|S_{s t}\right\|\left(\begin{array}{cc}
\frac{k-1}{k} & 0 \\
0 & k-1
\end{array}\right) .
$$

Using the same arguments that in the proof of Theorem 5.1, with $D(t), H(t)$ and $V(t)$ replaced by $U(s, t), \tilde{F}_{s t}$ and $\tilde{S}_{s t}$ respectively, formula (5) becomes

$$
\|U(s, t)\| M_{U(s, t)} \xi \cdot \xi=M_{F_{s t}} \xi \cdot \xi+M_{S_{s t}} \xi \cdot \xi+\epsilon(t),
$$

which, together with (5), concludes the proof of Theorem 5.2.

In concluding this section, we would like to refer to the recent paper by Capdeboscq and Kang [9] where it is shown that if the domain contains certain set of volume and is not thin than its PT stays away from the upper HS-bound.

It is also worth noticing that the asymptotic result in this section holds in the three-dimensional case as well. Indeed, taking combinations of ellipsoids, we can show by exactly the same arguments as those presented above the optimality of the upper bounds (1.1) and (1.2). 


\begin{tabular}{|c||c|c|c|c|}
\hline$\left(\lambda_{1}, \lambda_{2}\right)$ & $t^{*}$ & $s^{*}$ & $\left(\lambda_{1}\left(s^{*}, t^{*}\right), \lambda_{2}\left(s^{*}, t^{*}\right)\right)$ & $\frac{\left|\left(\lambda_{1}, \lambda_{2}\right)-\left(\lambda_{1}\left(s^{*}, t^{*}\right), \lambda_{2}\left(s^{*}, t^{*}\right)\right)\right|}{\left|\left(\lambda_{1}, \lambda_{2}\right)\right|}$ \\
\hline \hline$(1.0470,1.2893)$ & 9.5607 & 0.1992 & $(1.0480,1.2902)$ & $8.0838 \mathrm{E}-4$ \\
$(0.8277,1.4614)$ & 6.8182 & 0.6081 & $(0.8248,1.4586)$ & $2.3709 \mathrm{E}-3$ \\
$(1.2060,1.2060)$ & 14.4493 & $4.7730 \mathrm{E}-15$ & $(1.2062,1.2062)$ & $2.1222 \mathrm{E}-4$ \\
$(0.9387,1.5378)$ & 19.0739 & 0.4008 & $(0.9403,1.5394)$ & $1.2567 \mathrm{E}-3$ \\
$(0.7274,1.7702)$ & 18.4077 & 0.8089 & $(0.7253,1.7681)$ & $1.5114 \mathrm{E}-3$ \\
$(1.1015,1.4132)$ & 26.7781 & 0.1981 & $(1.1065,1.4182)$ & $3.9419 \mathrm{E}-3$ \\
\hline
\end{tabular}

TABLE 3. The numerical results for the identification algorithm. For a given eigenvalue pair $\left(\lambda_{1}, \lambda_{2}\right)$, we compute the parameters $t^{*}, s^{*}$ of the domain and find the eigenvalues $\left(\lambda_{1}\left(s^{*}, t^{*}\right), \lambda_{2}\left(s^{*}, t^{*}\right)\right)$. The last column is the relative error.

\section{IDENTIFICATION OF DOMAINS}

In this section, given a pair $\left(\lambda_{1}, \lambda_{2}\right)$ inside the HS bounds, we present a method to find the parameters $s$ and $t$, such that this pair arises as the eigenvalue pair of the PT associated with $U(s, t)$ or $L(s, t)$.

Our procedure is described in the following.

\section{Identification algorithm}

S'1. Given $\left(\lambda_{1}, \lambda_{2}\right) \in A_{1}$, transform it into

$$
\left(\rho_{1}, \rho_{2}\right):=V\left(\lambda_{1}, \lambda_{2}\right)
$$

S'2. Find the zero $t^{*} \geq 2$ satisfying

$$
P\left(\rho_{1}, t^{*}\right)=\rho_{2} .
$$

Here $P(x, t)$ is the polynomial computed in the previous section.

S'3. Compute $\left(\rho_{1}\left(s_{i}, t^{*}\right), \rho_{2}\left(s_{i}, t^{*}\right)\right)$ for $s_{i} \in S$. From these data, we derive the relation between $s$ and $\rho_{1}\left(s, t^{*}\right)$ as follows:

$$
x_{1}(s)=\sum_{i=0}^{m} \prod_{l \neq i}\left(\rho_{1}\left(s_{i}, t^{*}\right) \frac{s-s_{l}}{s_{i}-s_{l}}\right), \quad 0 \leq s \leq 1 .
$$

S'4. Find $s^{*}$ so that

$$
x_{1}\left(s^{*}\right)=\rho_{1} .
$$

We can check the validity of the above algorithm by computing the relative error. If $\left(s^{*}, t^{*}\right)$ is the computed pair of parameters, then we calculate the eigenvalues $\lambda_{1}\left(s^{*}, t^{*}\right)$ and $\lambda_{2}\left(s^{*}, t^{*}\right)$ of its associated PT. We can see the order of the relative error by computing

$$
\frac{\left|\left(\lambda_{1}, \lambda_{2}\right)-\left(\lambda_{1}\left(s^{*}, t^{*}\right), \lambda_{2}\left(s^{*}, t^{*}\right)\right)\right|}{\left|\left(\lambda_{1}, \lambda_{2}\right)\right|} .
$$

As shown in Table 3, the above algorithm identifies the parameterized domain pretty well. 


\section{Conclusion}

In this paper we proved that the PT associated with crosses approaches to the upper bound as cross gets thinner and longer. We then show, by numerical computations based on a boundary integral method, that every point inside the HashinShtrikman bounds for polarization tensors can be attained by a simply connected domain which is given explicitly in terms of two parameters. We also present a method to find the parameters corresponding to a given pair of eigenvalues inside the Hashin-Shtrikman bounds.

\section{ACKNOWLEDGEMENTS}

The question we consider in this paper was raised to us by Michael Vogelius. We acknowledge it with gratitude. H. Kang and E. Kim are partly supported by grant R02-2003-000-10012-0 from the Korea Science and Engineering Foundation. M. Lim is partly supported by the post-doctoral fellowship program of the Korea Science and Engineering Foundation.

\section{REFERENCES}

[1] H. Ammari and H. Kang, Reconstruction of Small Inhomogeneities from Boundary Measurements, Lecture Notes in Math. 1846, Springer-Verlag, Berlin, 2004.

$[2] \longrightarrow$, High-order terms in the asymptotic expansions of the steady-state voltage potentials in the presence of conductivity inhomogeneities of small diameter, SIAM J. Math. Anal., 34 (2003), pp. 1152-1166.

[3] - Properties of the generalized polarization tensors, Multiscale Modeling and Simulation: A SIAM Interdisciplinary Journal, 1 (2003), pp. 335-348.

[4] H. Ammari, H. Kang, and K. Kim, Anisotropic polarization tensors and effective properties of anisotropic composite materials, J. Diff. Equat., 215 (2005), pp. 401-428.

[5] H. Ammari, H. Kang, E. Kim, and M. Lim, Reconstruction of closely spaced small inclusions, SIAM J. Numer. Anal., 42 (2005), pp. 2408-2428.

[6] H. Ammari, H. Kang, and K. Touibi, Boundary layer techniques for deriving the effective properties of composite materials, Asymp. Anal. 41 (2005), pp. 119-140.

[7] E. Beretta, E. Francini, and M. S. Vogelius, Asymptotic formulas for steady state voltage potentials in the presence of thin inhomogeneities. A rigorous error analysis, J. Math. Pures Appl., 82 (2003), pp. 1277-1301.

[8] M. Brühl, M. Hanke, and M.S. Vogelius, A direct impedance tomography algorithm for locating small inhomogeneities, Numer. Math., 93 (2003), pp. 635-654.

[9] Y. Capdeboscq and H. Kang, Improved Hashin-Shtrikman bound for thick domains, Contemporary Mathematics, to appear.

[10] Y. Capdeboscq and M.S. Vogelius, A general representation formula for the boundary voltage perturbations casued by internal conductivity inhomogeneities of low volume fraction, Math. Modelling Num. Anal. 37 (2003), pp. 159-173.

[11] , Optimal asymptotic estimates for the volume of internal inhomogeneities in terms of multiple boundary measurements, Math. Modelling Num. Anal. 37 (2003), pp. 227240 .

[12] - A review of some recent work on impedance imaging for inhomogneieties of low volume fraction, to appear in Proceeding if the Pan-American Advanced Studies Institute on PDEs, Inverse problems and Nonlinear Analysis, January 2003. Contemporary Mathematics.

[13] D.J. Cedio-Fengya, S. Moskow, and M. Vogelius, Identification of conductivity imperfections of small diameter by boundary measurements: Continuous dependence and computational reconstruction, Inverse Problem, 14 (1998), pp. 553-595.

[14] G. Dassios and R.E. Kleinman, Low Frequency Scattering, Oxford Science Publications, The Clarendon Press, Oxford University Press, New York, 2000.

[15] V.V. Jikov, S.M. Kozlov, and O.A. Oleinik, Homogenization of Differential Operators and Integral Functionals, Springer-Verlag, Berlin, 1994. 
[16] R.E. Kleinman and T.B.A. Senior, Rayleigh scattering in Low and High Frequency Asymptotics, edited by V.K. Varadan and V.V. Varadan, North-Holland, 1986, pp. 1-70.

[17] R.V. Kohn and G.W. Milton, On bounding the effective conductivity of anisotropic composites, in Homogenization and Effective Moduli of Materials and Media, IMA Volumes in Mathematics and its Applications 1 (1986), pp. 97-125.

[18] R. Kress, Linear Integral Equations, Applied Mathematical Sciences, Vol. 82, Springer-Verlag, Berlin, 1980.

[19] R. Lipton, Inequalities for electric and elastic polarization tensors with applications to random composites, J. Mech. Phys. Solids, 41 (1993), pp. 809-833.

[20] G.W. Milton, The Theory of Composites, Cambridge Monographs on Applied and Computational Mathematics, Cambridge University Press, 2001.

[21] G. Pólya and G. Szegö, Isoperimetric Inequalities in Mathematical Physics, Ann. of Math. Stud. 27, Priceton University Pree, Priceton, NJ, 1951.

[22] M. Shiffer and G. Szegö, Virtual mass and polarization, Trans. Amer. Math. Soc., 67 (1949), pp. 130-205. 